\title{
Test lepton flavor universality with (semi)leptonic $D$ decays at BESIII
}

Sifan Zhang ${ }^{1,2^{*}}$ on behalf of the BESIII Collaboration

1 Nanjing University, Nanjing, China

2 Institute of High Energy Physics, Beijing, China

* zhangsf@ihep.ac.cn

November 12, 2018

Proceedings for the 15th International Workshop on Tau Lepton Physics, Amsterdam, The Netherlands, 24-28 September 2018

scipost.org/SciPostPhysProc. Tau2018

\section{Abstract}

Using $2.93 \mathrm{fb}^{-1}$ data taken at $\sqrt{s}=3.773 \mathrm{GeV}, 0.482 \mathrm{fb}^{-1}$ data taken at $\sqrt{s}=$ $4.009 \mathrm{GeV}$ and $3.19 \mathrm{fb}^{-1}$ data taken at $\sqrt{s}=4.178 \mathrm{GeV}$ with the BESIII detector, precision measurements of the branching fractions of $D^{0} \rightarrow K^{-} \mu^{+} \nu_{\mu}, D^{+} \rightarrow$ $\bar{K}^{0} \mu^{+} \nu_{\mu}, D^{0} \rightarrow \pi^{-} \mu^{+} \nu_{\mu}, D^{+} \rightarrow \pi^{0} \mu^{+} \nu_{\mu}, D^{+} \rightarrow \ell^{+} \nu_{\ell}$ and $D_{s}^{+} \rightarrow \ell^{+} \nu_{\ell}$ are performed. Combining the known branching fractions of $D \rightarrow \bar{K} e^{+} \nu_{e}, D \rightarrow \pi e^{+} \nu_{e}$ and $D_{s}^{+} \rightarrow$ $\tau^{+} \nu_{\tau}$, we have tested the lepton flavor universality with $D \rightarrow \bar{K} \ell^{+} \nu_{\ell}, D \rightarrow \pi \ell^{+} \nu_{\ell}$, $D^{+} \rightarrow \ell^{+} \nu_{\ell}$ and $D_{s}^{+} \rightarrow \ell^{+} \nu_{\ell}$ decays. We have also tested lepton flavor universality in different $q_{\ell \nu_{\ell}}^{2}$ intervals for $D \rightarrow \bar{K} \ell^{+} \nu_{\ell}(\ell=e$ or $\mu)$. Besides, hadronic form factor $f_{+}^{K}(0), D$ meson decay constant $f_{D_{s}^{+}}$and $f_{D^{+}}$, and quark mixing matrix element $\left|V_{c s}\right|$ and $\left|V_{c d}\right|$ are also extracted with the most precise accuracies to date.

\section{Contents}

1 Introduction 2

2 The BESIII detector and the analysis method 3

2.1 The BESWl detector 3

2.2 The analysis method 3

3 Recent results at BESIIII 3

$3.1 \quad$ Leptonic $D_{s}^{+}$decays 3

3.2 Leptonic $D^{+}$decays 5

3.3 Semileptonic $D^{0(+)}$ decays 5

$\begin{array}{lll}4 \text { Conclusion } & 7\end{array}$

$\begin{array}{lr}\text { References } & 8\end{array}$ 


\section{Introduction}

In the standard model (SM), the strong and weak interactions in leptonic and semileptonic $D$ decays can be well separated, as shown in Fig. $⿴$. The decay amplitude is described as the vector product of the hadronic current and the leptonic current. As a result, we
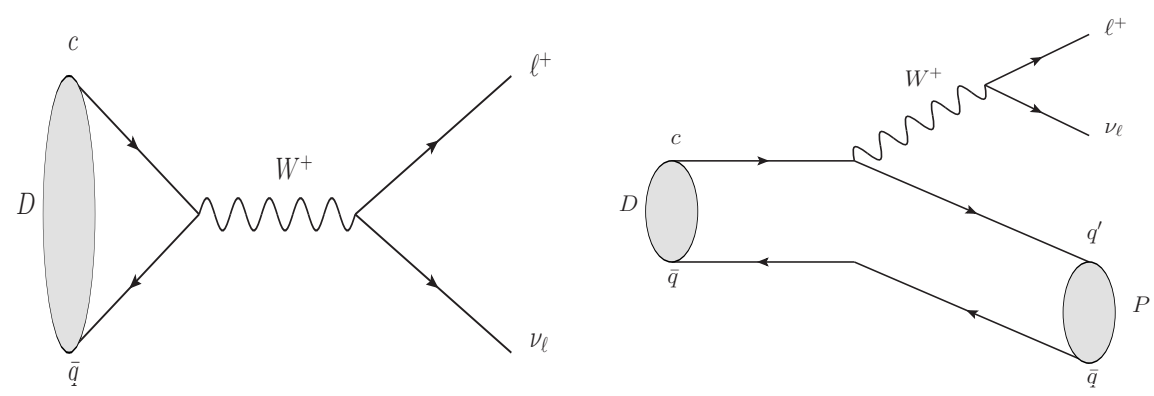

Figure 1: Feynman diagrams for leptonic $D$ decays (left) and semileptonic $D$ decays to pseudoscalar mesons (right).

have [I]

$$
\Gamma\left(D \rightarrow \ell \nu_{\ell}\right)=\frac{G_{F}^{2}}{8 \pi}\left(\left|V_{c s(d)}\right| f_{D}\right)^{2} m_{\ell}^{2} m_{D}\left(1-\frac{m_{\ell}^{2}}{m_{D_{s}^{+}}^{2}}\right)^{2}
$$

for pure leptonic decays, and

$$
\frac{d \Gamma\left(D \rightarrow P \ell \nu_{\ell}\right)}{d q^{2}}=\frac{G_{F}^{2}}{24 \pi^{3}}\left(\left|V_{c s(d)}\right| f_{+}\left(q^{2}\right)\right)^{2}\left|\vec{p}_{P}\right|^{3}+O\left(m_{\ell}^{2}\right)
$$

for semileptonic decays to pseudoscalar mesons, where $f_{D}$ and $f_{+}\left(q^{2}\right)$ are the $D$ meson decay constant and hadronic from factor parametrizing the hadronic current, $V_{c s(d)}$ is the Cabbibo-Kobayashi-Maskawa (CKM) matrix element describing the mixing between quark weak eigenstates and flavor eigenstates, $G_{F}$ is the Fermi coupling constant, $m_{D}$ and $m_{\ell}$ are the masses of $D$ meson and lepton [2], and $p_{P}$ is the momentum of the pseudoscalar meson.

Thus, if we calculate the ratio of the decay rates of (semi)leptonic decays involving different generations of leptons, the hadronic currents which are not well calculated in theory are almost canceled out. This gives a very strict constraint on the SM, which can be used to test the lepton flavor universality (LFU).

If there is a new physics (NP) mechanism that couples with different generations of leptons with different strengths, such as NP models involving a charged Higgs boson, or a NP mechanism that allows the lepton to change its flavor, such as the leptoquark mechanism, then the LFU in the SM may be violated [3-8]. Evidence of LFU violation has been found in $B$ meson decays in the measurements of $R_{D^{(*)}}=\frac{\Gamma\left(B \rightarrow D^{(*)} \tau^{+} \nu_{\tau}\right)}{\Gamma\left(B \rightarrow D^{(*)} \mu^{+} \nu_{\mu}\right)}$ and $R_{K^{(*)}}=\frac{\Gamma\left(B^{+} \rightarrow K^{(*)+} \mu^{+} \mu^{-}\right)}{\Gamma\left(B^{+} \rightarrow K^{(*)+} e^{+} e^{-}\right)}$[9-13]. While in the charm sector, no significant deviation from the SM prediction has been found [14]. With the world's largest $D \bar{D}$ samples near threshold, test of the LFU using (semi)leptonic $D$ decays with better precision at BESIII provides a chance to further test the SM and understand the anamolies in $B$ meson decays.

Besides, study of (semi)leptonic $D$ decays allow us to measure the corresponding $D$ meson decay constants, hadronic from factors, and the CKM matrix elements. These measurements can help to validate the theoretical calculations and test the unitarity of the CKM matrix. 


\section{The BESIII detector and the analysis method}

\subsection{The BESIII detector}

The BESIII detector is a magnitic spectrometer [15] located at the Beijing Electorn Positron Collider [16]. The cylindrical core of the BESIII detector consists of a heliumbased multilayer drift chamber, a plastic scintillator time-of-flight system (TOF), and a $\mathrm{CsI}(\mathrm{Tl})$ electromagnetic calorimeter (EMC), which are all enclosed in superconducting solenoidal magnet providing a $1.0 \mathrm{~T}$ magnetic field. The solenoid is supported by an octagonal flux-return yoke with resistive plate counter muon identifier modules interleaved with steel. The acceptance of charged particles and photons is $93 \%$ over $4 \pi$ solid angle. The charged-particle momentum resolution at $1 \mathrm{GeV} / c$ is $0.5 \%$, and the $d E / d x$ resolution is $6 \%$ for the electrons from Bhabha scattering. The EMC measures photon energies with a resolution of $2.5 \%(5 \%)$ at $1 \mathrm{GeV}$ in the barrel (end cap) region. The time resolution of the TOF barrel part is $68 \mathrm{ps}$, while that of the end cap part is $110 \mathrm{ps}$. The end cap TOF system is upgraded in 2015 with multi-gap resistive plate chamber technology, providing a time resolution of $60 \mathrm{ps}[\mathrm{[17}, 118]$.

\subsection{The analysis method}

For data taken at the center mass energy near $D \bar{D}$ threshold in $e^{+} e^{-}$collider, the $D$ mesons are produced in pair. If we fully reconstruct a $D$ meson via hadronic decays (called the single tagged $D$ meson), the other $D$ meson is guaranteed to exist. By searching for the (semi)leptonic decay in the recoiling system of the single tagged $D$ meson, we can then measure its branching fraction $(\mathrm{BF})$ via

$$
\mathcal{B}=\frac{N_{\text {sig }}}{N_{\mathrm{ST}} \varepsilon_{\text {sig }}}
$$

where $N_{\mathrm{ST}}$ and $N_{\text {sig }}$ are the number of single tagged $D$ mesons and the number of reconstructed (semi)leptonic events, respectively. $\varepsilon_{\text {sig }}$ is the efficiency of reconstructing the (semi)leptonic decay in the present of the single tagged $D$ meson. Here the number of reconstructed (semi)leptonic events is determined by examining the kinematic variables of the missing neutrio

$$
\begin{gathered}
U_{\text {miss }}=E_{\text {miss }}-\left|\vec{p}_{\text {miss }}\right| \\
M_{\text {miss }}^{2}=E_{\text {miss }}^{2}-\left|\vec{p}_{\text {miss }}\right|^{2}
\end{gathered}
$$

where $E_{\text {miss }}$ and $p_{\text {miss }}$ are the missing energy and missing momentum of the system. For correctly reconstructed (semi)leptonic decays, there variables should peak at zero.

\section{Recent results at BESIII}

\subsection{Leptonic $D_{s}^{+}$decays}

In 2014, BESIII collected $482 \mathrm{pb}^{-1}$ data at the center mass energy of $4.009 \mathrm{GeV}$ (near $D_{s}^{+} D_{s}^{-}$threshold. Using this data sample, the BFs of leptonic $D_{s}^{+}$decays are measured with a total number of $15127 \pm 321$ single tagged $D_{s}$ mesons reconstruced [1.9] . By constraining the ratio of the decay rates of $D_{s}^{+} \rightarrow \tau^{+} \nu_{\tau}$ and $D_{s}^{+} \rightarrow \mu^{+} \nu_{\mu}$ to the SM prediction 9.74, the BFs of these two decays are determined to be (See Fig. च)

$$
\mathcal{B}\left(D_{s}^{+} \rightarrow \mu^{+} \nu_{\mu}\right)=(0.495 \pm 0.067 \pm 0.026) \%,
$$




$$
\mathcal{B}\left(D_{s}^{+} \rightarrow \tau^{+} \nu_{\tau}\right)=(4.83 \pm 0.65 \pm 0.26) \% .
$$

With the input of $m_{D_{s}^{+}}$and $m_{\ell}$ from PDG, we have

$$
f_{D_{s}^{+}}\left|V_{c s}\right|=234.8 \pm 15.9 \pm 6.4 \mathrm{MeV} .
$$

The BFs are also determined without the SM constraint to be

$$
\begin{gathered}
\mathcal{B}\left(D_{s}^{+} \rightarrow \mu^{+} \nu_{\mu}\right)=(0.517 \pm 0.075 \pm 0.021) \%, \\
\mathcal{B}\left(D_{s}^{+} \rightarrow \tau^{+} \nu_{\tau}\right)=(3.28 \pm 1.83 \pm 0.37) \% .
\end{gathered}
$$

In 2016, BESIII collected another $3.19 \mathrm{fb}^{-1}$ data at the center mass energy of 4.178 $\mathrm{GeV}$. Taking advantage of the much higher $e^{+} e^{-} \rightarrow D_{s}^{+} D_{s}^{*-}$ cross section here, more precise measurements are perfromed with a total number of about $3.9 \times 10^{5}$ single tagged $D_{s}$ mesons reconstructed. The preliminary result of the $\mathrm{BF}$ of $D_{s}^{+} \rightarrow \mu^{+} \nu_{\mu}$ is (See Fig. [1])

$$
\mathcal{B}\left(D_{s}^{+} \rightarrow \mu^{+} \nu_{\mu}\right)=(0.528 \pm 0.015 \pm 0.014) \%,
$$

which gives

$$
f_{D_{s}^{+}}\left|V_{c s}\right|=242.5 \pm 3.5 \pm 3.7 \mathrm{MeV} .
$$

Combining with the $\mathrm{BF}$ of $D_{s}^{+} \rightarrow \tau^{+} \nu_{\tau}$ in PDG, we have

$$
R_{D_{s}^{+}}=\frac{\Gamma\left(D_{s}^{+} \rightarrow \tau^{+} \nu_{\tau}\right)}{\Gamma\left(D_{s}^{+} \rightarrow \mu^{+} \nu_{\mu}\right)}=10.19 \pm 0.52
$$

which is consistent with the SM prediction. The measurement of the BF of $D_{s}^{+} \rightarrow \tau^{+} \nu_{\tau}$ using this larger data sample is also ongoing.

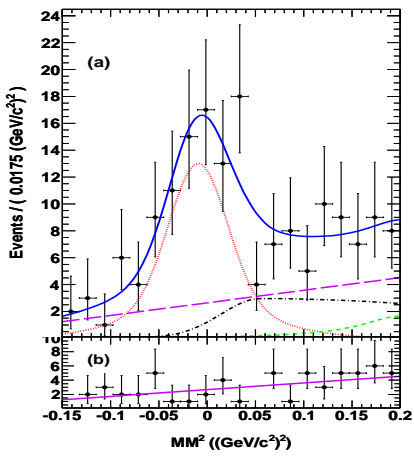

Figure 2: Projections of the simultaneous fit to the $M_{\text {miss }}^{2}$ distributions of events in (a) signal and (b) sideband region of the single tagged $D_{s}$ mesons. The red dotted curve shows the $\mu^{+} \nu_{\mu}$ signal and the black dotdashed curve shows the $\tau^{+} \nu_{\tau}$ signal.

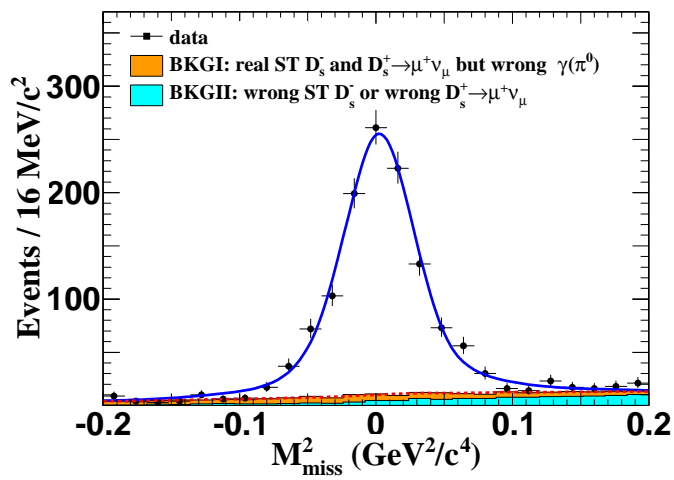

Figure 3: The fit to the $M_{\text {miss }}^{2}$ distribution of $D_{s}^{+} \rightarrow \mu^{+} \nu_{\mu}$ candidates. 


\subsection{Leptonic $D^{+}$decays}

Measurements of the BFs of leptonic $D^{+}$decays are perfromed using $2.93 \mathrm{fb}^{-} 1$ data at the center mass energy of $3.773 \mathrm{GeV}$ collected in 2010 and 2011 . With a total number of about $1.7 \times 10^{6}$ single tagged $D$ mesons reconstructed, the $\mathrm{BF}$ of $D^{+} \rightarrow \mu^{+} \nu_{\mu}$ is measured to be $(3.71 \pm 0.19 \pm 0.06) \times 10^{-4}$ (See Fig. 四) [20] , which gives

$$
f_{D^{+}}\left|V_{c d}\right|=45.75 \pm 1.20 \pm 0.39 \mathrm{MeV} .
$$

Meanwhile, preliminary result of the measurement of the BF of $D^{+} \rightarrow \tau^{+} \nu_{\tau}$ find the evidence of $D^{+} \rightarrow \tau^{+} \nu_{\tau}$ signal for the first time with a statistical significance of $4 \sigma$ (See Fig. (1) $)$. The $\mathrm{BF}$ is measured to be $(1.20 \pm 0.24) \times 10^{-3}$. Combining these two measurements we have

$$
R_{D^{+}}=\frac{\Gamma\left(D^{+} \rightarrow \tau^{+} \nu_{\tau}\right)}{\Gamma\left(D^{+} \rightarrow \mu^{+} \nu_{\mu}\right)}=3.21 \pm 0.64
$$

which is consistent with the SM prediction 2.66.
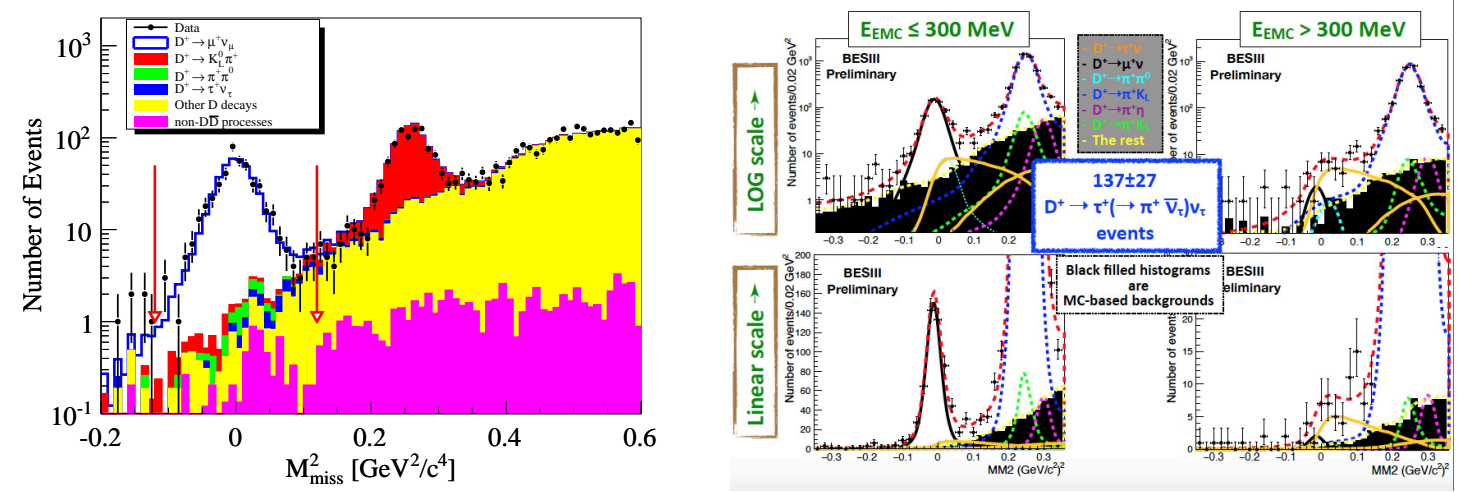

Figure 4: The fit to the $M_{\text {miss }}^{2}$ distribution Figure 5: The simultaneous fit to the $M_{\text {miss }}^{2}$ of $D^{+} \rightarrow \mu^{+} \nu_{\mu}$ candidates. distributions of the muon-like sample (with deposited energy in the EMC less than 300 $\mathrm{MeV}$ ) and the pion-like sample (with deposited energy in the EMC larger than 300 $\mathrm{MeV})$.

\subsection{Semileptonic $D^{0(+)}$ decays}

With the large $D \bar{D}$ sample at center mass energy of $3.773 \mathrm{GeV}$, BESIII has published the results of the BFs of $D \rightarrow \bar{K} e^{+} \nu_{e}$ and $D \rightarrow \pi e^{+} \nu_{e}$ with significantly improved precision [21-24]. However, further study in semileptonic $D$ decays concerning muon is still needed for more precise LFU test. In recent years, measurements of the BFs of these decays have been perfromed at BESIII. The BF $D^{0} \rightarrow K^{-} \mu^{+} \nu_{\mu}$ is measured to be $(3.413 \pm 0.019 \pm 0.035) \%$ (See Fig. 6) [25]. Combining with our previous measurement of $\mathcal{B}\left(D^{0} \rightarrow K^{-} e^{+} \nu_{e}\right)$, we have

$$
R_{K^{-}}=\frac{\Gamma\left(D^{0} \rightarrow K^{-} \mu^{+} \nu_{\mu}\right)}{\Gamma\left(D^{0} \rightarrow K^{-} e^{+} \nu_{e}\right)}=0.974 \pm 0.007 \pm 0.012 .
$$

With its largest statistics in semileptonic $D$ decays, measurements of hadronic from factor $f_{+}^{D \rightarrow K}(0)$ and CKM matrix element $\left|V_{c s}\right|$ are perfromed by a fit to the measured partial 
decay rates in separated $q^{2}$ intervals, which yields

$$
f_{+}^{D \rightarrow K}(0)\left|V_{c s}\right|=0.7133 \pm 0.0038 \pm 0.0029 .
$$

Meanwhile, LFU test is perfromed in various $q^{2}$ intervals and no significant deviation from SM prediction is found (See Fig. प).
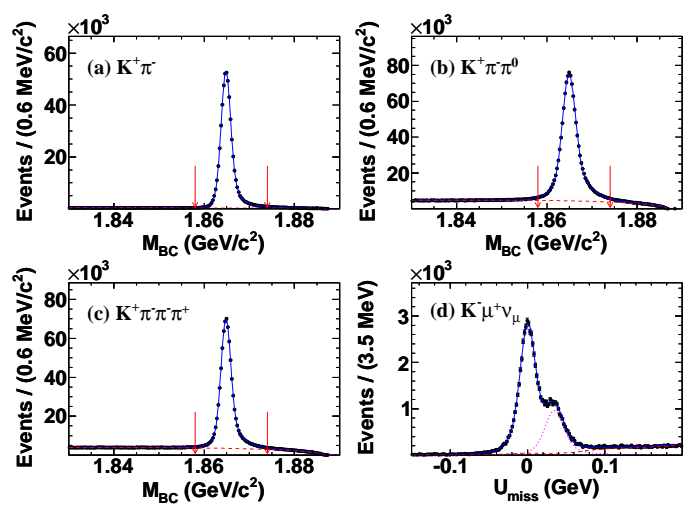

Figure 6: The fits to the mass distributions of single tagged $\mathrm{D}$ meons and to the $U_{\text {miss }}$ distribution of $D^{0} \rightarrow K^{-} \mu^{+} \nu_{\mu}$ candidates.
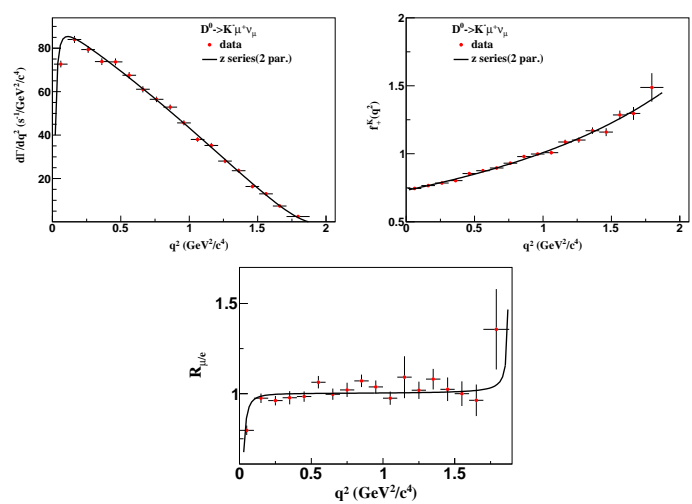

Figure 7: The fit to the partial decay rates of $D^{0} \rightarrow K^{-} \mu^{+} \nu_{\mu}$ (up left), the projection to the hadronic form factor (up right) and LFU test in various $q^{2}$ intervals (right).

The BF of $D^{+} \rightarrow \bar{K}^{0} \mu^{+} \nu_{\mu}$ is measured to be $(8.72 \pm 0.07 \pm 0.18) \%$ [26]. Here, the $\bar{K}^{0}$ meson is reconstructed via both $K_{S}^{0} \rightarrow \pi^{+} \pi^{-}$and $K_{S}^{0} \rightarrow \pi^{0} \pi^{0}$ decays and a simultaneous fit is perfromed for these two decays (See Fig. 8 ). Combining with our previous measurement, LFU test is perfromed with

$$
R_{\bar{K}^{0}}=\frac{\Gamma\left(D^{+} \rightarrow \bar{K}^{0} \mu^{+} \nu_{\mu}\right)}{\Gamma\left(D^{+} \rightarrow \bar{K}^{0} e^{+} \nu_{e}\right)}=0.988 \pm 0.033 .
$$

Measurements of the BFs of the Cabbibo suppressed decays $D^{0} \rightarrow \pi^{-} \mu^{+} \nu_{\mu}$ and $D^{+} \rightarrow$ $\pi^{0} \mu^{+} \nu_{\mu}$ are also perfromed at BESIII (See Fig. Q [) [27]. Combining our measured BFs

$$
\mathcal{B}\left(D^{0} \rightarrow \pi^{-} \mu^{+} \nu_{\mu}\right)=(0.272 \pm 0.008 \pm 0.006) \%
$$

and

$$
\mathcal{B}\left(D^{+} \rightarrow \pi^{0} \mu^{+} \nu_{\mu}\right)=(0.350 \pm 0.011 \pm 0.010) \%
$$

with our previous measurements yields

$$
R_{\pi^{-}}=\frac{\Gamma\left(D^{0} \rightarrow \pi^{-} \mu^{+} \nu_{\mu}\right)}{\Gamma\left(D^{0} \rightarrow \pi^{-} e^{+} \nu_{e}\right)}=0.922 \pm 0.030 \pm 0.022
$$

and

$$
R_{\pi^{0}}=\frac{\Gamma\left(D^{+} \rightarrow \pi^{0} \mu^{+} \nu_{\mu}\right)}{\Gamma\left(D^{+} \rightarrow \pi^{0} e^{+} \nu_{e}\right)}=0.964 \pm 0.037 \pm 0.026 .
$$




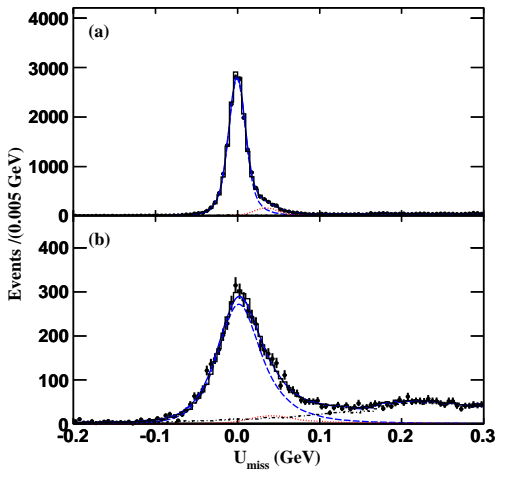

Figure 8: The simultaneous fit to the $U_{\text {miss }}$ distributions of $D^{+} \rightarrow \bar{K}^{0} \mu^{+} \nu_{\mu}$ candidates via (a) $K_{S}^{0} \rightarrow \pi^{+} \pi^{-}$and (b) $K_{S}^{0} \rightarrow \pi^{0} \pi^{0}$.

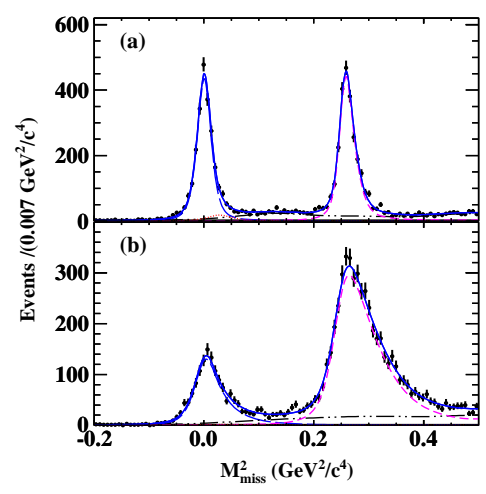

Figure 9: The fits to the $M_{\text {miss }}^{2}$ distributions of (a) $D^{0} \rightarrow \pi^{-} \mu^{+} \nu_{\mu}$ and (b) $D^{+} \rightarrow \pi^{0} \mu^{+} \nu_{\mu}$ candidates.

\section{Conclusion}

In summary, with the world's largest $D \bar{D}$ samples near threshold, precision measurements of the BFs of $D_{(s)}^{+} \rightarrow \ell^{+} \nu_{\ell}, D \rightarrow \bar{K} \mu^{+} \nu_{\mu}$ and $D \rightarrow \pi \mu^{+} \nu_{\mu}$ are performed at BESIII. Besides, CKM matrix elements $\left|V_{c s(d)}\right|, D$ meson decay constants $f_{D_{(s)}^{+}}$and hadronic form factor $f_{+}^{D \rightarrow K}(0)$ are extracted. With the input of $\left|V_{c s}\right|=0.97359_{-0.00011}^{+0.00010}[2],\left|V_{c d}\right|=$ $0.22438 \pm 0.00044[\bar{Z}], D_{s}^{+}$lifetime $\tau_{D_{s}^{+}}=(506.4 \pm 3.0 \pm 1.7 \pm 1.7) \times 10^{-15} \mathrm{~s}\left[[\bar{Z} 8], D^{+}\right.$lifetime $\tau_{D^{+}}=(1040 \pm 7) \times 10^{-15} \mathrm{~s} \mathrm{[Z]}, f_{D_{s}^{+}}=249.0 \pm 0.3_{-1.5}^{+1.1} \mathrm{MeV}$ [Z2. ],$f_{D^{+}}=212.6 \pm 0.4_{-1.2}^{+1.0}$ $\mathrm{MeV}$ [2.9] and $f_{+}^{D \rightarrow K}=0.747 \pm 0.011 \pm 0.015$ [30], we obtain

$$
f_{D_{s}^{+}}=252.3 \pm 3.7 \pm 3.6 \mathrm{MeV}
$$

and

$$
\left|V_{c s}\right|=0.974 \pm 0.014 \pm 0.016
$$

using leptonic $D_{s}^{+}$decays,

$$
f_{D^{+}}=203.9 \pm 5.3 \pm 1.8 \mathrm{MeV}
$$

and

$$
\left|V_{c d}\right|=0.2152 \pm 0.0056 \pm 0.0022
$$

using leptonic $D^{+}$decays,

$$
f_{+}^{D \rightarrow K}=0.7327 \pm 0.0039 \pm 0.0030
$$

and

$$
\left|V_{c s}\right|=0.955 \pm 0.006 \pm 0.024
$$

using $D^{0} \rightarrow K^{-} \mu^{+} \nu_{\mu}$ decay.

Meanwhile, LFU test using (semi)leptonic $D$ decays is perfromed at BESIII, and no significant deviation from the SM prediction is found at current statistics, as summarized in Table 田.

Funding information The author thanks for the support by Jonit Large-Scale Scientific Facility Funds of the National Natural Science Foundation of China and the Chinese Academy of Sciences under Contract No. U1532257. 
Table 1: LFU test at BESIII with (semi)leptonic $D$ decays.

\begin{tabular}{lcccccc}
\hline & $R\left(D_{s}^{+}\right)$ & $R\left(D^{+}\right)$ & $R\left(K^{-}\right)$ & $R\left(\bar{K}^{0}\right)$ & $R\left(\pi^{-}\right)$ & $R\left(\pi^{0}\right)$ \\
\hline SM & $9.74(1)$ & $2.66(1)$ & $0.975(1)[3 \mathrm{II}]$ & $0.975(1)[3 \mathrm{BI}]$ & $0.985(2)[$ [II $]$ & $0.985(2)[3 \mathrm{BI}]$ \\
BESIII & $10.19(52)$ & $3.21(64)$ & $0.978(14)$ & $0.988(33)$ & $0.922(37)$ & $0.964(45)$ \\
\hline
\end{tabular}

\section{References}

[1] D. Silverman and H. Yao, Relativistic treatment of light quarks in $D$ and $B$ mesons and $W$ exchange weak decays, Phys. Rev. D38, 214 (1988), doi:10.1103/PhysRevD.38.214.

[2] M. Tanabashi et al., Review of Particle Physics, Phys. Rev. D98, 030001 (2018), doi:10.1103/PhysRevD.98.030001.

[3] S. Fajfer, J. F. Kamenik and I. Nisandzic, On the $B \rightarrow D^{*} \tau \bar{\nu}_{\tau}$ sensitivity to new physics, Phys. Rev. D85, 094025 (2012), doi:10.1103/PhysRevD.85.094025.

[4] S. Fajfer, J. F. Kamenik, I. Nisandzic and J. Zupan, Implications of lepton flavor universality violations in B decays, Phys. Rev. Lett. 109, 161801 (2012), doi:10.1103/PhysRevLett.109.161801.

[5] A. Celis, M. Jung, X. Q. Li and A. Pich, Sensitivity to charged scalars in $B \rightarrow D^{(*)} \tau \nu_{\tau}$ and $B \rightarrow \tau \nu_{\tau}$ decays, JHEP 01, 054 (2013), doi:10.1007/JHEP01(2013)054.

[6] A. Crivellin, G. D'Ambrosio and J. Heeck, Explaining $h \rightarrow \mu^{ \pm} \tau^{\mp}, B \rightarrow K^{*} \mu^{+} \mu^{-}$and $B \rightarrow K \mu^{+} \mu^{-} / B \rightarrow K e^{+} e^{-}$in a two-Higgs-doublet model with gauged $L_{\mu}-L_{\tau}$, Phys. Rev. Lett. 114, 151801 (2015), doi:10.1103/PhysRevLett.114.151801.

[7] A. Crivellin, J. Heeck and P. Stoffer, A perturbed lepton-specific two-Higgs-doublet model facing experimental hints for physics beyond the Standard Model, Phys. Rev. Lett. 116, 081801 (2016), doi:10.1103/PhysRevLett.116.081801.

[8] M. Bauer and M. Neubert, Minimal leptoquark explanation for the $R_{D^{(*)}}$, $R_{K}$, and $(g-2)_{g}$ anomalies, Phys. Rev. Lett. 116, 141802 (2016), doi:10.1103/PhysRevLett.116.141802.

[9] J. P. Lee et al., Evidence for an excess of $\bar{B} \rightarrow D^{(*)} \tau^{-} \bar{\nu}_{\tau}$ decays, Phys. Rev. Lett. 109, 101802 (2012), doi:10.1103/PhysRevLett.109.101802.

[10] J. P. Lee et al., Measurement of an excess of $\bar{B} \rightarrow D^{(*)} \tau^{-} \bar{\nu}_{\tau}$ decays and implications for charged Higgs bosons, Phys. Rev. D88, 072012 (2013), doi:10.1103/PhysRevD.88.072012.

[11] R. Aaij et al., Measurement of the ratio of branching fractions $\mathcal{B}\left(\bar{B}^{0} \rightarrow D^{*+} \tau^{-} \bar{\nu}_{\tau}\right) / \mathcal{B}\left(\bar{B}^{0} \rightarrow D^{*+} \mu^{-} \bar{\nu}_{\mu}\right), \quad$ Phys. Rev. Lett. 115, 111803 (2015), doi:10.1103/PhysRevLett.115.159901, 10.1103/PhysRevLett.115.111803.

[12] R. Aaij et al., Test of lepton universality using $B^{+} \rightarrow K^{+} \ell^{+} \ell^{-}$decays, Phys. Rev. Lett. 113, 151601 (2014), doi:10.1103/PhysRevLett.113.151601.

[13] S. Wehle et al., Lepton-flavor-dependent angular analysis of $B \rightarrow K^{*} \ell^{+} \ell^{-}$, Phys. Rev. Lett. 118, 111801 (2017), doi:10.1103/PhysRevLett.118.111801. 
[14] S. Fajfer, I. Nisandzic and U. Rojec, Discerning new physics in charm meson leptonic and semileptonic decays, Phys. Rev. D91, 094009 (2015), doi:10.1103/PhysRevD.91.094009.

[15] M. Ablikim et al., Design and construction of the BESIII detector, Nucl. Instrum. Meth. A614, 345 (2010), doi:10.1016/j.nima.2009.12.050.

[16] C. H. Yu et al., BEPCII perfromance and beam dynamics studies on luminosity $\mathrm{p}$. TUYA01 (2016), doi:10.18429/JACoW-IPAC2016-TUYA01.

[17] X. Li et al., Study of MRPC technology for BESIII endcap-TOF upgrade, Radiation Detection Technology and Methods 1, 13 (2017), doi:10.1007/s41605-017-0014-2.

[18] Y. X. Guo et al., The study of time calibration for upgraded end cap TOF of BESIII, Radiation Detection Technology and Methods 1, 15 (2017), doi:10.1007/s41605-017(0)12-4.

[19] M. Ablikim et al., Measurement of the $D_{s}^{+} \rightarrow \ell^{+} \nu_{\ell}$ branching fractions and the decay constant $f_{D_{s}^{+}}$, Phys. Rev. D94, 072004 (2016), doi:10.1103/PhysRevD.94.072004.

[20] M. Ablikim et al., Precision measurements of $B\left(D^{+} \rightarrow \mu^{+} \nu_{\mu}\right)$, the pseudoscalar decay constant $f_{D^{+}}$, and the quark mixing matrix element $\left|V_{\mathrm{cd}}\right|$, Phys. Rev. D89, 051104 (2014), doi:10.1103/PhysRevD.89.051104.

[21] M. Ablikim et al., Study of decay dynamics and CP asymmetry in $D^{+} \rightarrow K_{L}^{0} e^{+} \nu_{e}$ decay, Phys. Rev. D92, 112008 (2015), doi:10.1103/PhysRevD.92.112008.

[22] M. Ablikim et al., Measurement of the absolute branching fraction of $D^{+} \rightarrow \bar{K}^{0} e^{+} \nu_{e}$ via $\bar{K}^{0} \rightarrow \pi^{0} \pi^{0}$, Chin. Phys. C40, 113001 (2016), doi:10.1088/1674$1137 / 40 / 11 / 113001$.

[23] M. Ablikim et al., Analysis of $D^{+} \rightarrow \bar{K}^{0} e^{+} \nu_{e}$ and $D^{+} \rightarrow \pi^{0} e^{+} \nu_{e}$ semileptonic decays, Phys. Rev. D96, 012002 (2017), doi:10.1103/PhysRevD.96.012002.

[24] M. Ablikim et al., Study of dynamics of $D^{0} \rightarrow K^{-} e^{+} \nu_{e}$ and $D^{0} \rightarrow \pi^{-} e^{+} \nu_{e}$ decays, Phys. Rev. D92, 072012 (2015), doi:10.1103/PhysRevD.92.072012.

[25] M. Ablikim et al., Study of the $D^{0} \rightarrow K^{-} \mu^{+} \nu_{\mu}$ dynamics and test of lepton flavor universality with $D^{0} \rightarrow K^{-} \ell^{+} \nu_{\ell}$ decays, http://arxiv.org/abs/1810.03127.

[26] M. Ablikim et al., Improved measurement of the absolute branching fraction of $D^{+} \rightarrow \bar{K}^{0} \mu^{+} \nu_{\mu}$, Eur. Phys. J. C76, 369 (2016), doi:10.1140/epjc/s10052-016-4198-2.

[27] M. Ablikim et al., Measurement of the branching fraction for the semi-leptonic decay $D^{0(+)} \rightarrow \pi^{-(0)} \mu^{+} \nu_{\mu}$ and test of lepton universality, Phys. Rev. Lett. 121, 171803 (2018), doi:10.1103/PhysRevLett.121.171803.

[28] R. Aaij et al., Measurement of $B_{s}^{0}$ and $D_{s}^{-}$meson lifetimes, Phys. Rev. Lett. 119, 101801 (2017), doi:10.1103/PhysRevLett.119.101801.

[29] A. Bazavov et al., Charmed and light pseudoscalar meson decay constants from four-flavor lattice QCD with physical light quarks, Phys. Rev. D90, 074509 (2014), doi:10.1103/PhysRevD.90.074509.

[30] H. Na, C. T. H. Davies, E. Follana, G. P. Lepage and J. Shigemitsu, The $D \rightarrow K, \ell \nu$ semileptonic decay scalar from factor and $\left|V_{c s}\right|$ from Lattice $Q C D$, Phys. Rev. D82, 114506 (2010), doi:10.1103/PhysRevD.82.114506. 
[31] L. Riggio, G. Salerno and S. Simula, Extraction of $\left|V_{c d}\right|$ and $V_{c s} \mid$ from experimental decay rates using lattice $Q C D D \rightarrow \pi(K) \ell \nu$ form facotrs, Eur. Phys. J. C78, 501 (2018), doi:10.1140/epjc/s10052-018-5943-5. 